\title{
The Feasibility Study of Transporting Wireless CDMA Signals Over HFC Networks
}

\author{
Yu-Min Lin and Winston I. Way \\ Department of Communications Engineering \\ National Chiao-Tung University \\ Hsinchu, Taiwan, Republic of China \\ Fax:886-3-5718870 Phone:886-3-5729138 E-mail:wiway@cc.nctu.edu.tw
}

\begin{abstract}
Wireless access in hybrid fiber/coax (HFC) networks can integrate two major telecommunication infrastructure, i.e., wireless and HFC networks. This paper studies the technical feasibility of integrating IS-95 code-division-multiple-access (CDMA) wireless networks and HFC networks. Capacity estimation of an HFC optical node-based CDMA cellular systems using a return-path channel model is provided. In addition to multiple access interference in IS-95 CDMA networks, the returnpath channel model takes into account ingress noise, impulsive noise, and micro-reflections in a coaxial cable transmission system, and laser diode clipping-induced intermodulation noise in an optical fiber link. Furthermore, in our Monte Carlo simulation, we have assumed slow flat fading for radio propagation, and ideal timing recovery and power control.
\end{abstract}

\section{INTRODUCTION}

Today's CATV networks are enjoying a high penetration rate in many countries. Consequently, HFC networks, which is essentially a two-way CATV network with optical fiber trunks and feeders, are bound to play an important role in bringing interactive broadband services to homes and business areas. Parallel to this HFC network evolution process is the miniaturization of cell size in today's cellular wireless networks to increase network capacity. In the mean time, personal communication service (PCS) networks are also on the horizon. IS-95 CDMA voice-application-oriented systems, in particular, have been standardized by EIA/TIA ${ }^{\prime}$, and have been or will soon be operated in many countries around the world, such as United States, South Korea, China, Japan, Israel, etc. CDMA technique is also inherently immune to narrow band interference, which exists in HFC systems, and can be used in future wideband applications (i.e., IMT-2000) ${ }^{2}$.

The purpose of this paper is to study whether the HFC network infrastructure can be utilized to provide IS-95 CDMA services, especially to accommodate the increasing number of radio antenna ports (RAPs) for wireless access. RAPs can be installed in an HFC network as shown in Fig.l (a) or 1(b), respectively. In Fig.1 (a), each RAP is installed in an optical node where a downstream optical 
receiver and an upstream laser transmitter reside, and the RAP is at the center of a microcell. In this case, both upstream and downstream wireless signals are transported only through optical fiber links. Various systems considerations and upstream optical transmitter circuit designs to meet the required dynamic range requirements in a microcell have been discussed ${ }^{3-5}$. On the other hand, Fig.l (b) is applicable to situations such as (1) to extend cell coverage or to resolve a "blind-zone" coverage problem in Fig.1 (a), and (2) to increase resource sharing in low-traffic service areas (all RAPs use the same radio spectrum and call processing equipment $)^{6-9}$. However, the technical feasibility and design criteria of the transmission systems given in Fig.l (b), which has all RAPs installed in the coaxial cable feeders or taps, have not yet been studied thoroughly. In this paper, we choose to investigate the return-path transmission systems, i.e., from the RAPs to the CATV headend. In particular, we want to study how well IS-95 CDMA signals can tolerate the abundant ingress and impulse noise sources in the return-path spectrum (5 to $40 \mathrm{MHz}$ in North American CATV Systems), and how the return-path-laserclipping induced intermodulation noise may affect the IS-95 signals.

The organization of this paper is as follows. In section 2, the overall system simulation block diagram with a review of IS-95 wireless CDMA system is presented. Various HFC transmission impairments, which may degrade the overall system performance, are described in section 3. The capacity estimations via Monte Carlo computer simulation, considering the various impairments given in section 3, are presented in section 4. The conclusion is given in Section 5 .

\section{OVERALL SYSTEM SIMULATION BLOCK DIAGRAM}

The overall system simulation block diagram is given in Fig.2. It consists of a pair of IS-95 CDMA uplink transmitter and receiver, multiple access interference inherent to CDMA systems, and a channel model which separately considers various system impairments including ingress noise, impulse noise, and micro-reflections in coaxial systems, in addition to a return-path clipped-laser model for the return path. The impairment factors of a coaxial cable system will be described in section 3. The uplink IS-95 CDMA system is briefly reviewed as follows.

\subsection{Uplink IS-95 CDMA System}

As shown in Fig. $2^{10}$, the $9.6 \mathrm{kbps}$ baseband data is first sent to a rate- $1 / 3$ constraint length- 9 convolutional encoder, and then to a block interleaver with parameters $32 \times 18$, to obtain an output symbol rate of $28.8 \mathrm{ksps}$. Subsequently, a 64 -ary orthogonal modulation is performed by selecting a row (out of 64 rows) of 64-bit Walsh code indexed by every six incoming data bits (with 64 possible combinations). At this point, the symbol rate becomes $307.2 \mathrm{ksps}(=28.8 / 6 \times 64)$. The coded symbol is then spread and scrambled, for the purpose of privacy, by mixing with the $1.2288 \mathrm{Mbps}$ long code (period $2^{42}-1$ ) from the long code generator. The scrambled data stream is spread further by simultaneously spreading the data stream in quadrature with short-length (period $2^{15}$ ) sequences. The 
resulting quadrature channels are applied to an offset-QPSK modulator for $\mathrm{I} / \mathrm{Q}$ filtering and mixing up to the carrier frequency. The receiver part of an IS-95 uplink is just the reverse of the transmitter, except that there is an additional RAKE receiver to achieve a diversity improvement on a frequencyselective fading channel, which is normally encountered in a cellular network environment. The RAKE receiver is not modeled in our computer simulation because we assume flat frequency fading for the radio propagation, and because a RAKE receiver cannot resolve any micro-reflection-induced multipath delay less than about $1 \mu \mathrm{s}$. Note that typical delays among micro-reflection-induced multipaths in a coaxial system are less than a few hundred nanoseconds ${ }^{11}$.

\section{IMPARIMENT FACTORS IN HFC SYSTEMS}

\subsection{Impulse Noise (duration $<<1 \mathrm{~ms}$ )}

Man-made impulse noise can easily be coupled into the coaxial part of an HFC network from the home environment. For example, motor ignition, and the turn-on and turn-off of electric facilities are the annoying sources. Other sources such as corona discharges from power lines, and discharges across corroded connector contacts can also cause a bursty noise in the network. Our measurement show that the time duration of this kind of noise is about 2 4 microseconds, as illustrated in Fig.3.

\subsection{Ingress Noise (duration $>1 \mathrm{~ms}$ )}

Ingress noise includes narrowband short-wave signals, ham radio, paging signals, etc. We have measured the return-path ingress noise of a typical CATV system, and the results are shown in Fig.4. We can see that the ingress noise in the spectrum below $20 \mathrm{MHz}$ is relatively high.

\subsection{Multipath Interference}

The impedance mismatch of connectors, taps, splitters and amplifiers contribute to the reflections in the upstream path. Unlike wireless environment, delays due to coaxial cable reflections are short, often less than a few hundred nanoseconds.

\subsection{Return-Path Laser Clipping-Induced Intermodulation Noise}

If the return-path spectrum between 5 and $40 \mathrm{MHz}$ is to accommodate a number of frequency-divisionmultiplexed IS-95 CDMA signals, it is possible that these signals can generate intermodulation beats because of the nonlinear characteristics of a return-path laser. Furthermore, if the optical modulation index per channel is to be maximized in order to increase the available power budget, the intermodulation noise will be dominated by laser-clipping induced intermodulation noise. 


\section{THEORETICAL CAPACITY OF SPREAD SPECTRUM 64-ARY ORTHOGONAL MODULATION}

The capacity of spread spectrum 64-ary orthogonal modulation (from point A to B in Fig.2) was theoretically investigated ${ }^{12}$. According to that report, the multiple-access-interference-limited bit error rate (BER) is given by

$$
P_{b}=\left(\frac{2^{k-1}}{2^{k}-1}\right) \sum_{n=1}^{M}(-1)^{n+1}\left(\begin{array}{c}
M-1 \\
n
\end{array}\right) \frac{1}{n+1} \exp \left(-\frac{n k E_{b}}{(n+1) N_{1}}\right)
$$

Where

$$
N_{t}=\frac{N_{0}}{2}+\frac{k E_{b}}{6 L}\left(2 N_{u}-1\right)
$$

In Eqs. (1) and (2), $N_{o}=$ White noise spectral density, $E_{b}=B$ it energy, $N_{u}=$ Number of users, $L=256, M=64$, and $k=6$. The calculated results based on Eqs (1) and (2) are shown in Fig.5. We can see that when the received $\left(E_{b} / N_{o}\right)_{r}$ (the ratio of bit energy to white noise spectral density) per user measured at point $\mathrm{B}$ in Fig. 2 is increased from 8 to $\sim 22 \mathrm{~dB}$, there is a significant improvement in the BER performance for the same number of users. However, when $\left(E_{b} / N_{o}\right)_{r}$ per user is increased beyond $\sim 22 d B$, negligible BER improvement can be obtained. Therefore, for the spread spectrum 64-ary orthogonal modulation system shown in Fig.2 (point $A$ to $B$ ), we can set the minimum required $\left(E_{b} / N_{o}\right)_{r}$ per user to be $22 \mathrm{~dB}$.

\section{COMPUTER SIMULATION RESULTS}

The computer simulation approach that we used is based on Monte Carlo method. The BER is directly computed from time-domain decisions. To ensure that our computer simulation is correct, we checked our simulation results with those obtained from Section 4, as shown in Fig.5. It can be seen that the results from computer simulation match perfectly with those from theoretical calculations. We then use our Monte Carlo simulation to study the IS-95 capacity for various channel impairments mentioned in Section 3 (with simulation parameters following the standard IS-95 specifications).

\subsection{IS-95 Capacity Degradation Due to Multiple Access Interference}

The estimated capacity which considers only added white gaussian noise (AWGN) and multipleaccess-interference (MAI) is shown in Fig.6. The typical BER requirement for voice transmission is $10^{-3}$. Under this criterion, IS-95 can have at most 32 users simultaneously on line in a single CDMA bandwidth, i.e., $1.25 \mathrm{MHz}$, at $\left(\mathrm{E}_{\mathrm{b}} / \mathrm{N}_{\mathrm{o}}\right)_{\mathrm{r}}$ per user $\geq 22 \mathrm{~dB}$. 
For an IS-95 uplink, the commonly cited criterion is $\left(\mathrm{E}_{\mathrm{b}} /\left(\mathrm{N}_{\mathrm{o}}+\mathrm{I}_{\mathrm{o}}\right)\right)_{\mathrm{r}}$ per user $=6 \mathrm{~dB}^{10}$. Since the processing gain in an IS-95 uplink is $128(=1.2288 \mathrm{Mbps} / 9.6 \mathrm{kbps})$ or $\sim 21 \mathrm{~dB}$, the required $\left(\mathrm{E}_{\mathrm{b}} / \mathrm{I}_{\mathrm{o}}\right)_{\mathrm{r}}$ per user before despreading can be as low as $-15 \mathrm{~dB}$, when MAI dominates over white noise ${ }^{13,14}$. However, $\left(\mathrm{E}_{\mathrm{b}} / \mathrm{I}_{\mathrm{o}}\right)_{\mathrm{r}}=-15 \mathrm{~dB}$ is equivalent to a condition with about 31 interfering users. Therefore, the total user number is 32. This is quite consistent with our simulation results given in Fig.6.

\subsection{IS-95 Capacity Degradation Due to Impulse Noise}

We model the impulse noise as a Poisson process. The interval of each impulse is uniformly distributed between 2 to 4 microseconds. The Poisson process arrival rate is 200 (per second). The noise power is calculated as a normalized power with respect to the signal power. The capacity reduction due to impulse noise is shown in Fig.7. We observe that the effect of a bursty noise with such a short duration only reduces the IS-95 capacity from 32 to 29, even when the bursty noise power is $\sim 30 \mathrm{~dB}$ higher than that of the signal power. The reason is that the Viterbi decoder of the uplink IS95 system, which has a free distance of 18 , is very powerful, and the short impulse noise can only cause $\sim 2$ symbol errors (for a symbol rate of $1.2288 \mathrm{Mbps}$ ).

\subsection{IS-95 Capacity Degradation Due to Ingress Noise}

The simulation results of capacity reduction as a function of the ratio of ingress noise power and signal power is shown in Fig.8. The results show that the CDMA system capacity gradually reduces when the ingress noise power increases from -2 to $+9 \mathrm{~dB}$ relative to the signal power. The capacity reduces only $20 \%$ when the ingress noise power is above the signal power by $7.5 \mathrm{~dB}$. In contrast, in wireless systems using TDMA or FDMA multiple access schemes, this ingress noise level can shut down the entire link. The main reason is that CDMA can spread the narrowband noise in the receiver, which results in a less damaging white-noise-like interference.

\subsection{IS-95 Capacity Degradation Due to Multipath Interference}

In the coaxial cable part of HFC systems, relative delays due to multiple reflections is less than $1 \mu s$. In our computer simulation, we model a "strong reflection" case ${ }^{11}$ which has a second-path signal whose power is $13 \mathrm{~dB}$ lower than that of the main path, and has a delay of 600 nanoseconds with respect to the main path. The results are shown in Fig. 6, from which we can see that only two more users are lost due to strong reflection.

\subsection{Laser Clipping Noise}

The clipping phenomenon in a return-path laser ${ }^{15}$ induces intermodulation noise and can set an upper bound of the optical modulation index (OMI) of the CDMA signals. We assume that the laser has an ideal output light power versus bias current characteristics (L-I curve), as shown in Fig.2. The simulation results are shown in Fig. 9 for single channel, and Fig. 10 for 17 channels of IS-95 signals, 
respectively. The 17 channels (each occupying a $2 \mathrm{MHz}$ bandwidth, including guard bands) can fully occupy the 5 to $40 \mathrm{MHz}$ return path band of an HFC system. Both figures show the degradation of $\left(E_{b} / N_{o}\right)_{r}\left(=\left(E_{b} / N_{o}\right)_{r, \text { in }}-\left(E_{b} / N_{o}\right)_{r, o u t}\right)$ and the resultant capacity as a function of the rms OMI/channel, where $\left(E_{b} / N_{o}\right)_{r, \text { in }}$ and $\left(E_{b} / N_{o}\right)_{r, \text { out }}$ represent the $\left(E_{b} / N_{o}\right)_{r}$ at the input and output of a clipped-laser diode, respectively. Since the clipping-induced in-band intermodulation noise is gaussian-like, which cannot be despread by the IS- 95 receiver, we can see that both $\left(\mathrm{E}_{\mathrm{b}} / \mathrm{N}_{\mathrm{o}}\right)_{\text {rout }}$ and the corresponding capacity decrease quickly as $\mathrm{OMI} / \mathrm{channel}$ increases. If $10 \%$ capacity reduction is allowed, the $\mathrm{rms} \mathrm{OMI} / \mathrm{ch}$ for 17 IS-95 channels should be kept below 0.26 . If no capacity reduction is allowed, the rms $\mathrm{OMI} / \mathrm{ch}$ for single and 17 IS-95 channels should be kept below 0.55 and 0.13 , respectively.

\section{CONCLUSION}

The feasibility of integrating wireless IS-95 CDMA systems and HFC networks is studied in this paper. Various impairments in HFC return-path are discussed and their effects on wireless link capacity are estimated through computer simulation. We observe that the short bursty noise normally encountered in HFC systems does not seriously affect the capacity of IS-95 systems, thanks to the powerful deinterleaver and Viterbi decoder. When considering ingress noise, we note that when there is a strong in-band narrowband noise, the IS-95 system capacity does decrease, but unlike the case in TDMA and FDMA systems, IS-95 systems can still support some active users. RAKE receiver can not be utilized to cope with the short multipath delay due to micro-reflections in coaxial cables, so micro-reflection can contribute to a small capacity reduction. Finally, the clipping-induced intermodulation noise has to be carefully minimized by controlling the OMI/ch of each IS-95 signal. When no capacity degradation is allowed in the return-path laser, the rms OMI/ch for single and 17 IS-95 channels should be kept below 0.55 and 0.13 , respectively. These numbers may have to be further reduced if a sufficient system margin is to be reserved.

\section{Reference}

1. TIA/EIA/IS-95 "Mobile Station-Base Station Compatibility Standard for Dual-Mode Wideband Spread Spectrum Cellular System," Telecommunication Industry Association, July 1993.

2. P. W. Baier, P. Jung, A. Klein, "Taking the challenge of multiple access for third-generation cellular mobile radio system - a European view," IEEE Comm. Magazine, vol. 34, pp. 82-89, Feb 1996.

3. W. I. Way, "Optical fiber-based microcullar systems: an overview," IEICE Trans. on Communications, vol. E76-B, pp. 1091-1102, September 1993.

4. D. M. Curter, J. B. Georges, T. H. Le, and K. Y. Lau, "Dynamic range requirements for optical transmitters in fiber-fed microcellular networks," IEEE Photo. Technology, vol. 43, pp.564-566, May 1995. 
5. W. I. Way, Y. K. Yen, W. W. Deng, "Super-FM and analog-to-digital conversion circuit techniques for wireless access," IEE J. Quantum Electronics, September 1996.

6. R. W. Donaldson and A. S. Beasley, "Wireless CATV network access for personal communications using simulcasting," IEEE Trans. on. Vehicular Technology, vol. 43, pp. 666-671, August 1994.

7. N. K. Shankaranarayanan, et al., "Multiport wireless access system using fiber/coax networks for personal communication services (PCS) and subscriber loop applications," Proc. IEEE Globecomm'95, Singapore, Nov. 1995.

8. K. J. Kerpez, “A radio access with distributed antennas," IEEE Trans. on Vehicular Tech., vol. 45, pp. 265-275, May 1996.

9. S. Ariyavisitakul, T. E. Darcie, L. J. Greenstein, M. R. Philips, and N. K. Shankaranarayanan. "Performance of simulcast wireless techniques for personal communication systems," IEEE J. on Selected Areas in Comm., vol. 14, pp. 632-642, May 1996.

10. R. L. Peterson, R. W. Ziemer, D. E. Borth, Introduction to spread spectrum communications, pp. 536, Prentice Hall, 1995.

11. K. Laudel, E. Tsui, J. Harp, A. Chun, and J. Robinson, " Performance of a 256-QAM demodulator/equalizer in a cable environment," Technical Papers, pp. 283-304, 43th NCTA Annual Convention and Exposition, Technical Papers, New Orleans, 1994.

12. H. Zhang and D. Rutkowski, "Performance analysis of a spread spectrum M-ary orthogonal modulation under multipath Fading," Singapore ICCS'94 Conference Proceedings, pp. 602-606, vol. 2.

13. K. S. Gilhousen, I. M. Jacobs, R. Padovani, A. J. Viterbi, L. A. Weaver, Jr., and C. E. Wheatley, III, "On the capacity of a cellular CDMA system," IEEE Trans. on Vehicular Tech., vol. 40, May 1991.

14. R. Padovani, "Reverse link performance of IS-95 based cellular systems," IEEE Personal Communication, Third Quarter 1994.

15. P. Y. Chiang and W. I. Way, "Ultimate capacity of a laser diode in transporting multichannel MQAM signals,” J. on Lightwave Tech., vol. 15, pp. 1914-1924, October 1997. 


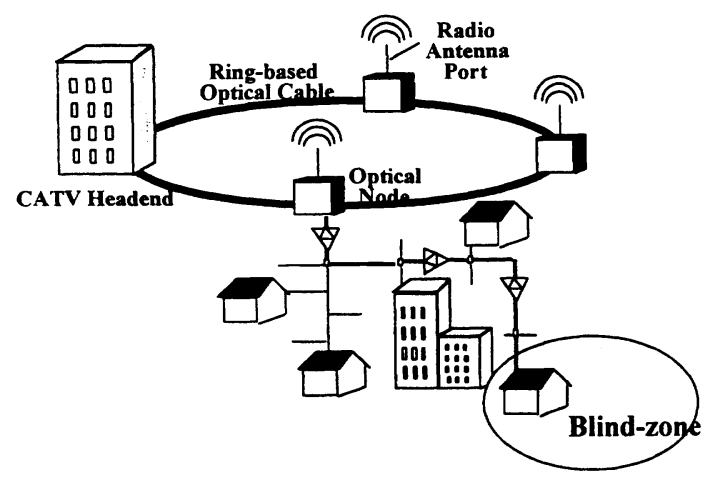

(a)

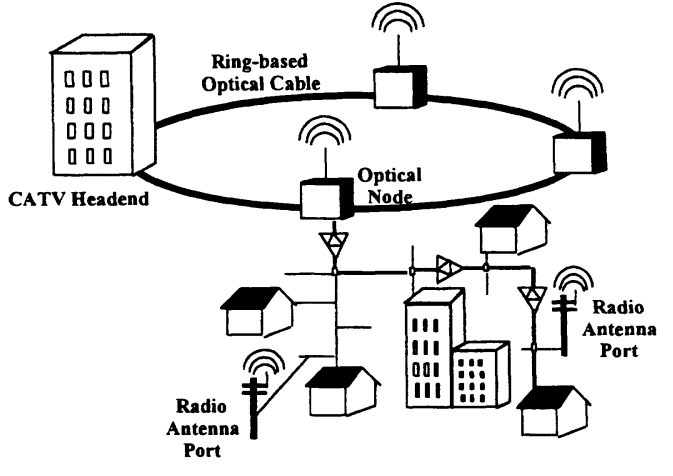

(b)

Figure 1. Wireless access in HFC system architecture

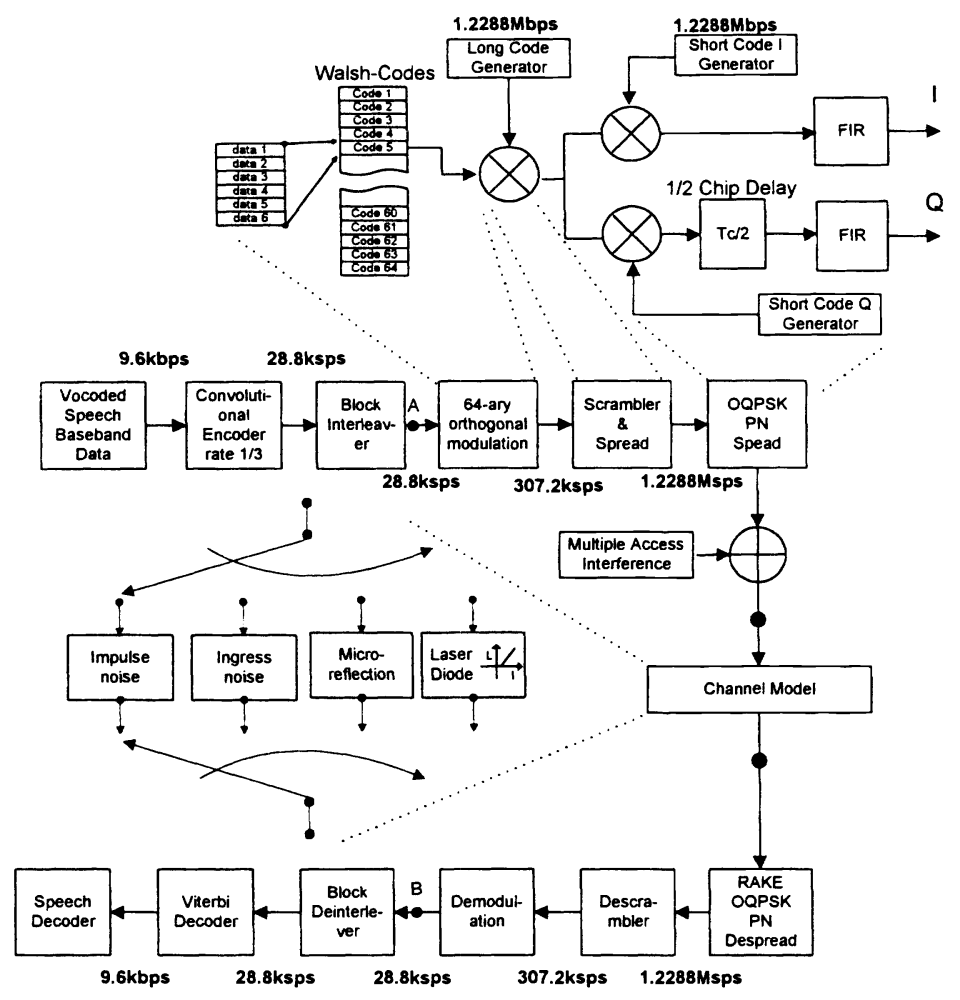

Figure 2. Uplink IS-95 block diagram 


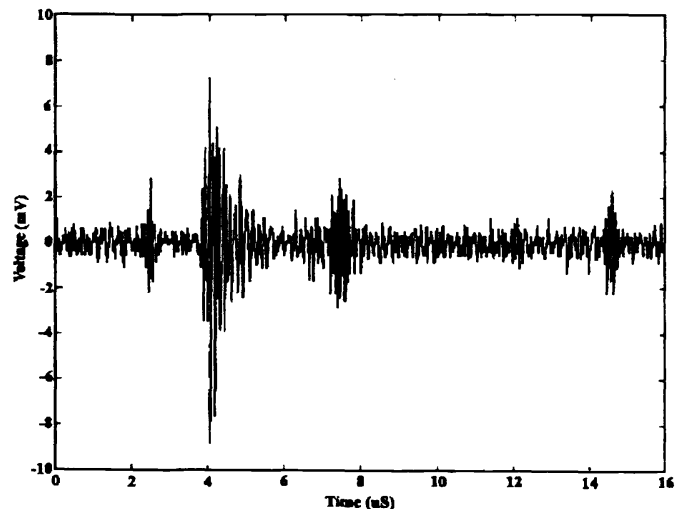

Figure 3. Typical impulse noise time-domain waveform. In this case, the impulse noise was measured from a Hsinchu-city CATV network, when a home vaccum cleaner was turned on.

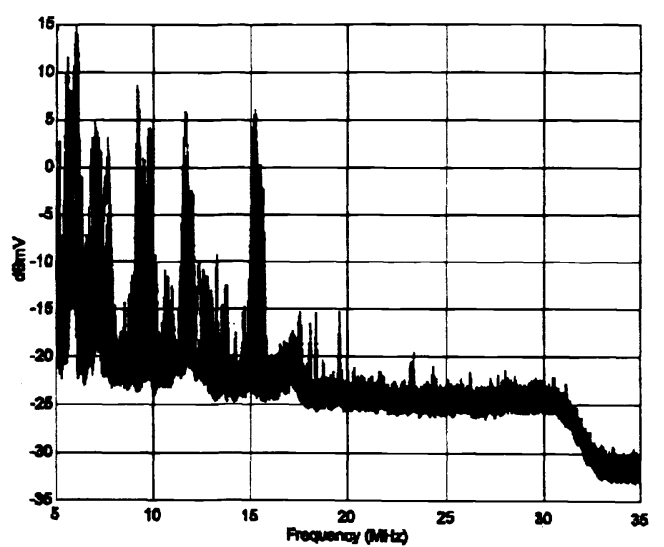

Figure 4. Ingress noise spectrum of an HFC return-path. Data was measured over 24 hours from a Hsinchu-city CATV network.

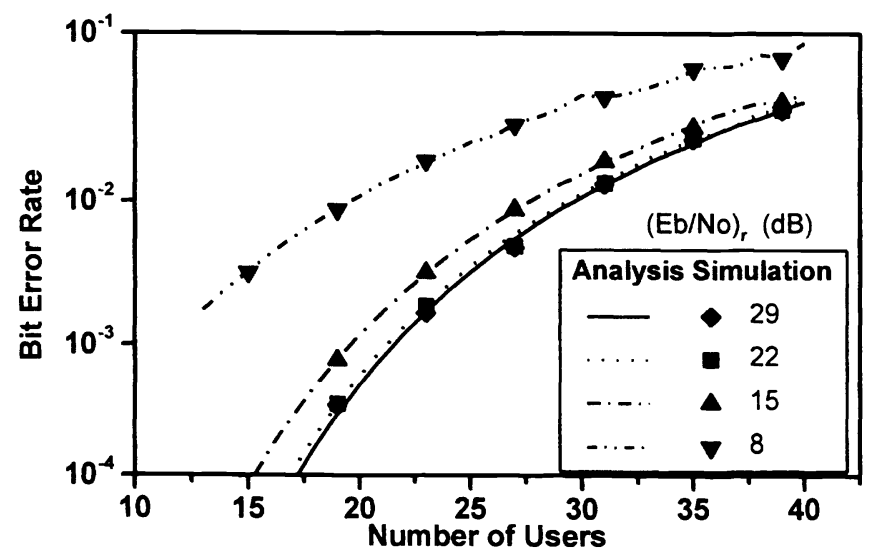

Figure 5. Performance of 64-ary modulation with rectangular pulse 


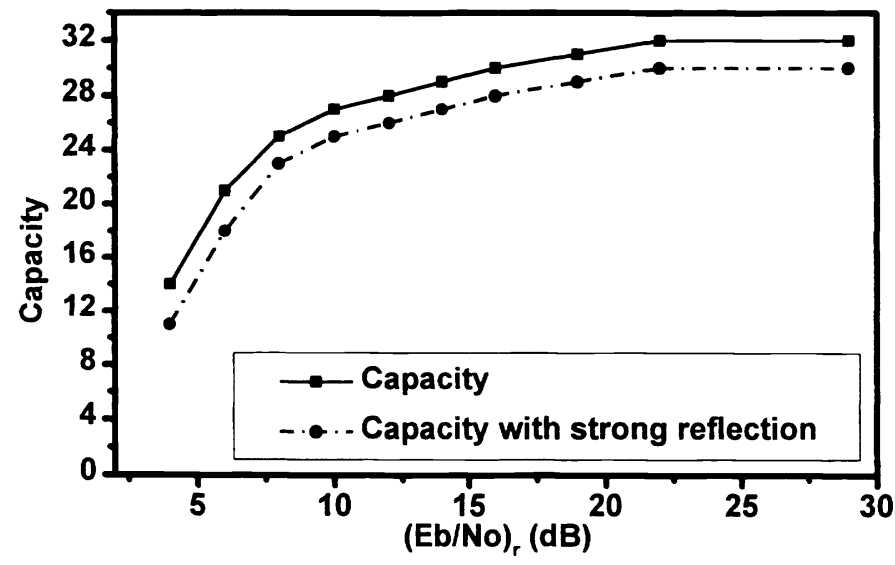

Figure 6. IS-95 Capacity $\left(\mathrm{BER}=10^{-3}\right)$ as a function of $(\mathrm{Eb} / \mathrm{No}) \mathrm{r} w$ and w/o microreflection
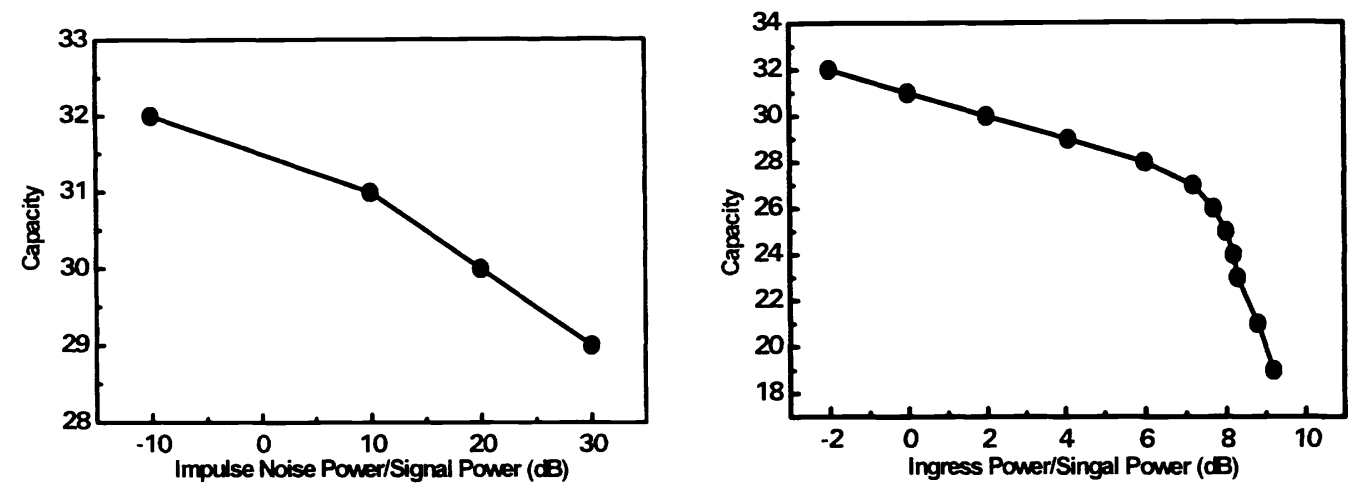

Figure 7. Effect of impulse noise on IS-95 capacity

Figure 8. Effect of ingress noise on IS-95 capacity

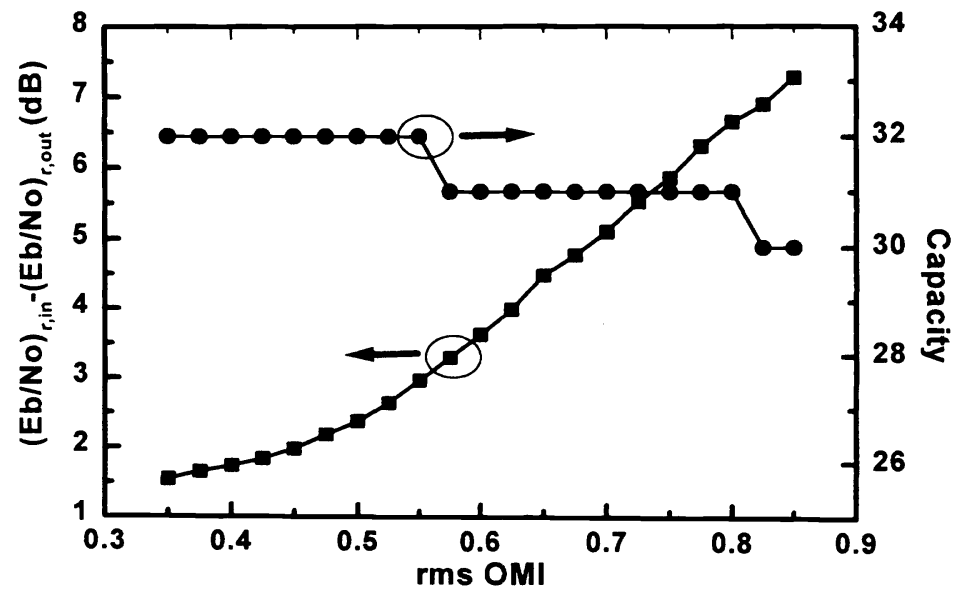

Figure 9. (Eb/No)r reduction and capacity as a function of rms OMI for single IS-96 channel 


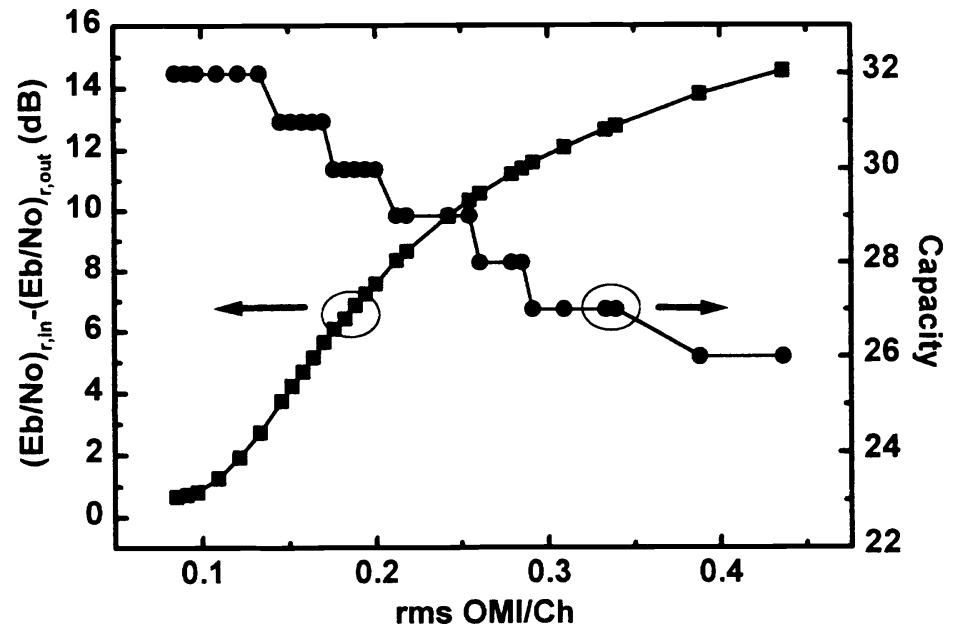

Figure 10. (Eb/No)r reduction and capacity as a function of $\mathrm{rms} \mathrm{OMI/Ch}$ for 17-channel IS-95 signal 